\title{
Predicting the Resiliency in Parents with Exceptional Children Based on Their Mindfulness
}

\author{
Sosan Jabbari ${ }^{1}$, Somayeh Sadati Firoozabadi ${ }^{1} \&$ Sedighe Rostami $^{2}$ \\ ${ }^{1}$ Faculty Education and Psychology, Department of Education of Special children, Shiraz University, Shiraz, \\ Iran \\ ${ }^{2}$ Psychology and Educational of Exceptional Children, Shiraz University, Shiraz, Iran \\ Correspondence: Sedighe Rostami, Shiraz University, Shiraz, Iran. Tel: 98-0917-718-6039. E-mail: \\ sedigherostami2014@gmail.com
}

Received: August 1, 2016

Accepted: September 20, 2016

Online Published: October 31, 2016

doi:10.5539/jel.v5n4p285

URL: http://dx.doi.org/10.5539/jel.v5n4p285

\begin{abstract}
The purpose of the present study was to predict the resiliency in parents with exceptional children based on their mindfulness. This descriptive correlational study was performed on 260 parents of student (105 male and 159 female) that were selected by cluster sampling method. Family resiliency questionnaire (Sickby, 2005) and five aspect mindfulness questionnaire were used as measurement instruments. Data were analyzed using descriptive statistics, pearson correlation coefficient and inter regression showed some dimension of predicted.
\end{abstract}

Keywords: resiliency, mindfulness, exceptional children

\section{Introduction}

Having a disabled child in family affects the structure and mental health of family members especially the parents (Bardon, 1980). These parents are generally exposed to the danger of different life problems (Beckman, 1991). Parents of exceptional children usually face various stressors and mental and social pressures and compared to other parents, experience more stress, depression and other problems (King, 1999).

Exceptional children need the special help of the family and parents are ones who have the most relationships and interactions with them. Caring and training these children can be effective on quality of life in these individuals. A collection of these factors, imposes great psychological pressure on parents of these children that affects all dimensions of life and the whole structure of the family (Kaveh, 2009). The health of these parents is placed in the second degree of importance because of the stressing needs of the children and the heavy duty of caring those (Saif Naraghi \& Nadi, 2012). Therefore, parents of these children have lower physical and mental health and show more distress. This distress is continued in all stages of life and is also added on. These problems affect the health and mental hygiene of family and society. Therefore, parents of these children need more attention and support (Kaniskan, 2008). Considering the mental problems of parents with exceptional children, resiliency is one of the factors of adaptation and promotion of mental health. Resiliency is one of the variables that is related to the mental vulnerability. Resiliency is considered as a concepts of positive psychology and refers to the process of dynamism of positive adaptation with sad and tragic experiences (Musten, 2001). Resiliency is a factor that helps individuals facing and adapting difficult and stressful life condition and protects them of mental disorders and life problems (Izadian, 2010). Tolerant individuals have high personal adaptation with environmental stressful factors in their life (Musen \& Reid, 2004). Also, resiliency is defined as the skills and abilities that enable individual to adapt with difficulties and problems (Alword, 2005).

Several factors can affect higher resiliency in individuals especially parents of children with exceptional children. These factors include emotional intelligence, positive and negative emotions, personality, the level of stress and mindfulness (Jasin, 2011; Shine, 2004; Sasagava \& Fryke, 2006). In recent years, psychology has been affected of various concepts that one of them is mindfulness. Mindfulness is one of the ways of reducing mental pressure and can be explained as a way of "being" or a way of understanding that needs understanding personal emotions (Boer, 2003).

Mindfulness includes full flexibility of the attention to the experiences of the present time with a state of acceptance and without any judgment (Kabat-zin, 1990). These experiences include humane thoughts and 
emotions that are accepted just as immediate transient phenomena and opposite to cognitive-behavioral methods without any need to content analysis or changing them. Individuals with mindfulness understand internal and external realities freely and without distortion and have a lot of abilities facing a wide range of thoughts, emotions and experiences. Mindfulness is typically described as an attentive, munjudmenta, and receptive awarencess of present moment experience in term of feeling, images, thought's and sensations, perceptions (e.g., Kabat-Zinn, 1990). According to Bishop et al. (2004) mindfulness has two main components:

(a) the Volitional regulation of attention and (b) the adoption of an orientation toward present moment experience characterized by dispassionate curiosity openness to what is occurring, and acceptance.

Increasing mindfulness is associated with increasing the psychological well-being. Researches have shown that individuals with mindfulness are more able in identifying, managing and solving the daily problems (Zeidan, 2010; Walker \& Kulusimo, 2011; Siegel, 2010).

Cumpfer (1999) resulted in a study that positive adaptation with life can cause higher level of resiliency. Individuals with high resiliency achieve high psychological health. Lotzering (2005) showed tolerant individuals do not have self-break behaviors, are emotionally relieved and have the ability to cope with unpleasant situation. Himmani (2002) examined the resiliency and conquering future problems in families with children with learning disability or physical disability in a study and showed the parents of these children need adaptation and supportive patterns.

Siegel (2010) believes that mindfulness practices are useful for a significant range of individuals with various problems such as reduction of life problems, depression, sadness and grief. Bear et al. (2004) examined the relationship between mindfulness and psychological well-being in a study. The results showed there is a positive significant relationship between mindfulness and psychological well-being and a negative relationship with stress, anxiety and depression.

Nicklick (2011) trained mindfulness skills to a group of anxious students. The results indicated that this training has useful effects on reducing the level of anxiety and symptoms of pain. Generally, some researches show that mindfulness training is effective on reducing aggression, obsessive thoughts and anxiety and treating behavioral and emotional problems of children with growth insufficiency (Hwang Corney, 2013; Syne et al., 2003).

Therefore, mindfulness can be considered as a mediator for increasing psychological performance and reducing the symptoms of stress that can increase the resiliency in families. Therefore, considering the breadth of the subject and the researches that were mentioned and few researches that have been conducted in case of the relationship between the desired variables in parents with exceptional children, the present study aims to examine the role of mindfulness and resiliency in families and answer the question whether dimensions of mindfulness has the power to predict the significance of resiliency or not.

\section{Methods}

The present study was a correlational study. To analyze the data, SPSS-21 software and descriptive statistic (mean and standard deviation) and multidimensional regression. Parents of exceptional students in Shiraz in academic year of 2015-2016 consisted the statistical population of the study. The sample consisted 260 parents of students (105 male students and 159 female students) that were selected by cluster sampling method. Among elementary and middle schools of Shiraz, 6 special schools with students having various disorders (autism, blind, deaf, mentally retarded and behavioral disorders) were selected randomly. The questionnaires were given to the parents and they were asked to complete two questionnaire related to family resiliency and cognitive regulation of emotion based on the guideline of each questionnaire. In the present study, Family Resiliency Questionnaire and Mindfulness Questionnaire were used.

\section{Family Resiliency Questionnaire}

This questionnaire was used in order to measure family resiliency. This scale is a standard tool to measure resiliency that is designed by Sickby (2005) based on Family resiliency systematic theory by Walsh (2003) this scare is culture free. This tool is a 4-degree Likert scale from 1 (totally disagree) to 4 (totally agree). This questionnaire has 66 items with Likert range that examines the level of family resiliency in 6 fields including family relationship, problem solving, taking benefit of economic and social resources, keeping a positive point of view, family ties, spirituality in family and the ability to develop meaning for difficulties. The minimum score of this scale is 66 and the maximum score is 204. High score in this questionnaire shows that the family has the high level of resiliency and low score in this questionnaire shows that the family has the low level of resiliency. Psychometric evidence of this scale is confirmed by Sickby (2005). Sickby has examined the reliability of this tool using Chronbach's alpha and examined its validity using construct validity, predicting validity, criterion 
validity and simultaneous validity. Also, Bouchanan (2008) reported the reliability of whole scale and reported Chronbach's alpha of 0.93 . In the present study, the reliability of the questionnaire was examined Chronbach's alpha that was 0.85 .

\section{Five-Aspect Mindfulness Questionnaire}

This scale is a 39-item self-report questionnaire that is developed by Baer et al. (2006) this scare is culture free, through integrating items of Mindfulness Questionnaire by Freeberg, Consciousness and Mindfulness Attention Scale, Curiosity Mindfulness Scale, Revised Scale of Cognitive and Emotional Mindfulness and Sonamiton Mindfulness Questionnaire using factor analysis. The internal consistency of the factors of the questionnaire was good and alpha coefficient for not being reactive is 0.75 , description is 0.91 , observation is 0.83 , act with consciousness is 0.87 , and non-randomness is 0.87 . The correlation between factors was average, although it was statistically significant and was in a range from 0.15 to 0.34 . Baer et al. (2006) measured Five-aspect Mindfulness Questionnaire with constructs such as emotional intelligence, openness, extraversion, neuroticism, psychological symptoms, suppression of thought, difficulty in emotion regulation, Alexey Time, experiential avoidance. The results showed there is positive relationship between mindfulness, openness and emotional intelligence and negative relationship between Alexey Time mindfulness, psychological symptoms, neuroticism and suppression of thought. They did regression analysis and concluded that psychological symptoms can be predicted by aspects of mindfulness. Also, they examined the construct validity of Five-aspect Mindfulness Questionnaire in two groups, one of them with the experience of meditation and the other one without the experience of meditation. The range of the scores was 39-195 in this scale. A total score is resulted from the sum of the scores in each subscale that show the more the score, the higher the mindfulness. Subscales include observation, description, act with consciousness, not judging and not reacting. In the present study, the validity of the questionnaire is examined using Chronbach's alpha that its coefficient is 0.92 for the whole scale.

\section{Findings}

The descriptive findings of the variables of the study include mean and standard deviation. Table 1 shows these indices.

Table 1. Mean and standard deviation of dimensions of mindfulness and resiliency

\begin{tabular}{lcc}
\hline Dimensions & Mean & Standard deviation \\
\hline resiliency & 188.80 & 19.87 \\
observing & 26.33 & 4.85 \\
describing & 21.92 & 3.52 \\
acting with awareness & 21.83 & 4.92 \\
Non-judging & 23.56 & 4.08 \\
Non-reactivity & 20.37 & 3.69 \\
mindfulness & 116.73 & 12.18 \\
\hline
\end{tabular}

As it is seen in table, the results showed that among mindfulness dimensions, the observation has higher mean (26.32) and not reacting has the lowest mean (20.37).

Pearson correlation coefficient was used to investigate the relationship between mindfulness dimensions and resiliency (Table 2). As shown in Table 2, there were significant positive correlation between observing, describing, non-reactivity and resiliency $(\mathrm{p}<0.01)$. There was a negative correlation between acting with awareness and resiliency $(\mathrm{p}<0.01)$. Acting with awareness had the maximum correlation with resiliency $(\mathrm{r}=-0.42)$. 
Table 2. Correlation between dimensions of mindfulness and resiliency

\begin{tabular}{cccccc}
\hline variables & observing & describing & Acting with awareness & Non judging & Non reactivity \\
\hline resiliency & $0.20^{* *}$ & $0.23^{* *}$ & $-0.42^{* *}$ & 0.04 & $0.35^{* *}$ \\
\hline
\end{tabular}

$* * \mathrm{p}<0.01$

To examine the power of each mindfulness dimension to predict resiliency in family, multiple regression analysis was used. The results are shown in Table 3.

Table 3. Summary of the model of the regression of variance analysis and statistical feature of resiliency based on mindfulness

\begin{tabular}{|c|c|c|c|c|c|c|c|c|c|}
\hline Predictor variables & $\begin{array}{l}\text { Criterion } \\
\text { variable }\end{array}$ & $\mathrm{R}$ & $\mathrm{R}^{2}$ & $\mathrm{~F}$ & $\mathrm{p}<0.0001$ & $\mathrm{~B}$ & $\beta$ & $\mathrm{t}$ & $\mathrm{p}<0.001$ \\
\hline observing & & & & & & 0.19 & 0.05 & 0.78 & - \\
\hline describing & & 0.57 & 0.33 & 24.81 & 0.0001 & 0.72 & 0.13 & 2.07 & 0.039 \\
\hline acting with awareness & resiliency & & & & & -1.72 & -0.43 & -8.02 & 0.0001 \\
\hline Non-judging & & & & & & 0.29 & 0.06 & 1.06 & - \\
\hline Non-reactivity & & & & & & 1.57 & 0.29 & 5.32 & 0.0001 \\
\hline
\end{tabular}

Multiple regression analysis was performed to deeper investigate the relationship between mindfulness dimensions and resiliency. The results are shown in Table 3. Describing, acting with awareness and non-reactivity dimensions were able to estimate resiliency.

\section{Conclusion}

The results of multivariable regression analysis in this study showed there is a relationship between mindfulness and resiliency. In the present study, among dimensions of mindfulness, dimensions of description, not reacting and act with consciousness have a significant relationship with resiliency. Description and not reacting dimensions predicted resiliency positively and act with consciousness predicted resiliency negatively. This finding was consistent with the results of studies by Siegel (2010); Bear et al. (2004); Tabrizchi and Heidari (2015); Jefri et al. (2015); and Prosmin (2008).

To explain this finding, it can be said that optimal resiliency can reduce mental pressure and anxiety potentially. Acquiring this skill can enable the individual to stop or limit showing emotional or judgmental reactions to the desired subject and respond to issues in life with more consciousness. Based on the previous researches, it can be said about mindfulness that it can have significant positive effect on parents in case of mental pressure, anxiety, coping strategies, etc. (Ver \& Tomic, 2009). Mindfulness interventions have been used with parents for the prevention and treatment of mental health problems; however, until recently, MBSR specifically has not been used to address parental stress (Bazzano et al., 2013; Neece, 2013). Findings indicate that general mindfulness practices are effective in reducing parental stress, rumination, and reactivity, while decreasing dysfunctional parenting habits as well as improving the broader family environment, including marital function and co-parenting (Bogels, Lehtonen, \& Restifo, 2010).

Since mindfulness strengthens both mental and behavioral performance (Chung et al., 2004), it can be expected that individuals with mindfulness have more positive toward their abilities and act successfully in coping mental pressure of life. It was found in a study that was conducted on parents of children with autism that mindfulness-based strategies may be the only choice for those parents whose children need cognitively or behaviorally. Mindfulness teaches the individuals how become more aware of themselves and their reactions and how to attend the present time without judging. These methods causes the development of more adaptive responses facing the mental pressure of having a child with special need. It can in turn increase resiliency in these parents, avoid developing negative emotions in these individuals and cause the parents with exceptional children to experience higher resiliency. Considering the findings, it is recommended mindfulness-based stress reduction program be used to improve health in these families. A growing body of research suggests that Mindfulness Based Stress Reduction (MBSR) is an effective intervention enhance adaptive coping techniques. 
The practice of mind fullness requires the development of an international state of moment-to-moment awareness of internal and external stimuli in a non-judgmental fashion (Baer, 2003; Hayes, 2011), allowing observation to be dealt with as needed, putting the mindfulness practitioner in the position of choosing how to respond rather than being in a constant state of reactivity, mindfulness base stress reduction is a specific manualized mindfulness intervention program supported by over two decades of extensive research showing its effectiveness in reducing stress, anxiety and depression, and promoting overall wellbeing (Chiesa \& Serretti, 2009).

Results of our study must be viewed in the light methodological limitation small sample sizes with a medium to high education level of parents, although results are encouraging they must be interpreted within the context of several study limitation the age and education of parents.

Future research should include a program for training mindfulness. Also focus to variables such as age and social economic and level of education of parent. Recommendations for future research in light of the results of the study the researcher recommends that it would be beneficial to replicate this study on other groups of parents also it would be helpful to have more participants from mothers and fathers in order to be able to provide more generalizable.

\section{References}

Baer, R. (2006). Mindfulness Training as a clinical intervention: A conceptual and empirical review. Clinical psychology: Science and practice, 10(2), 125-143. http://dx.doi.org/ 10.1093/clipsy.bpg015

Baer, R. A. (2003). Mindfulness Training as a Clinical Intervention: A Conceptual and Empirical Review. Clinical Psychology: Science and Practice, 10(2), 125-143. http://dx.doi.org/10.1093/clipsy/bpg015

Bazzano, A., Wolfe, C., Zylowska, L., Wang, S., Schuster, E., Barrett, C., \& Lehrer, D. (2013). Mindfulness Based Stress Reduction (MBSR) for Parents and Caregivers of Individuals with Developmental Disabilities: A Community-Based Approach. Journal of Child and Family Studies, 24(2), 298-308. http://dx.doi.org/10.1007/s10826-013-9836-9

Beckman, P. J. (1991). Comparison of mothers and fathers perception of the effect of young children with and without disabilities. American Journal on Mental Retardation, 95, 585-595.

Bishop, S. R., Lau, M., Shapiro, S., Carlson, L., Anderson, N. D., Carmody, J., ... Devins, G. (2004). Mindfulness: A Proposed Operational Definition. Clinical Psychology: Science and Practice, 11(3), 230-241. http://dx.doi.org/10.1093/clipsy.bph077

Bogar, C. B., \& Hulse-Killacky, D. (2006). Resiliency Determinants and Resiliency Processes among Female Adult Survivors of Childhood Sexual Abuse. Journal of Counseling \& Development, 84(3), 318-327. http://dx.doi.org/ 10.1002/j.1556-6678.2006.tb00411

Bögels, S. M., Lehtonen, A., \& Restifo, K. (2010). Mindful Parenting in Mental Health Care. Mindfulness, 1(2), 107-120. http://dx.doi.org/10.1007/s12671-010-0014-5

Burden, R. L. (1980). Measuring the effects of stress on the mothers of handicapped infants: Must depression always follow? Child: Care, Health and Development, 6(2), 111-125. http://dx.doi.org/ 10.1111/j.1365-2214.1980.tb00803.x

Carmody, J., \& Baer, R. A. (2007). Relationships between mindfulness practice and levels of mindfulness, medical and psychological symptoms and well-being in a mindfulness-based stress reduction program. $J$ Behav Med, 31(1), 23-33. http://dx.doi.org/ 10.1007/s10865-007-9130-7

Chang, V. Y., Palesh, O., Caldwell, R., Glasgow, N., Abramson, M., Luskin, F., \& Koopman, C. (2004). The effects of a mindfulness-based stress reduction program on stress, mindfulness self-efficacy, and positive states of mind. Stress and Health, 20(3), 141-147. http://dx.doi.org/ 10.1002/smi.1011

Chiesa, A., \& Serretti, A. (2009). Mindfulness-Based Stress Reduction for Stress Management in Healthy People: A Review and Meta-Analysis. The Journal of Alternative and Complementary Medicine, 15(5), 593-600. http://dx.doi.org/10.1089/acm.2008.0495

Heimani, T. (2002). Parents of children with disabilities: Resilience, coping and future Expectation. Journal of Developmental and Physical Disabilities, 14(2), 159-171. http://dx.doi.org/10.1023/A:1015219514621

Hollis-Walker, L., \& Colosimo, K. (2011). Mindfulness, self-compassion, and happiness in non-meditators: A theoretical and empirical examination. Personality and Individual Differences, 50(2), 222-227. http://dx.doi.org/10.1016/j.paid.2010.09.033 
Izadian, N., Amiri, M., Jahromi, R., \& Hamidi, S. H. (2010). A study of relationship between suicidal ideas, depression, resiliency, daily stresses and mental health among Tehran university students. Procedia Social and Behavioral Sciences, 5, 1515-1519.

Jeffety, D., \& Austern, S. (2015). Deafness and Challenging Behaviour: The $360^{\circ}$ Perspective. Wachington D.C.

Kabat-Zinn, J. (1990). Full catastrophe living: Using the wisdom of your body and mind to face stress, pain and illness. New York: Dell publishing.

Kabat-Zinn, J. (2013). Full catastrophe living: Using the wisdom of your body and mind to face stress, pain, and illness.

Kaniskan, R. B., Ortega, \& Beauchemin, A. (2008). Best practices in mental health. Lyeeum Books, 4(1).

Keng, S. L. (2011). Effects of mindfulness on psychological health: A review of empirical studies. Clinical Psychology Review, 31(6), 1041-1056. http://dx.doi.org/ 10.1016/j.cpr.2011.04.006

Kumpfer, K. L. (1999). Factor and processes contributing to resilience framework. In M. D. Glants, \& J. L. Johnson (Eds.), Resilince and development (pp. 179-224). New York.

Latzihing, T. D., Block, J., \& Funder, D. C. (2005). Ego-control and ego-resiliency: Generalization of self-report scales based on personality descriptions from acquaintances, clinicians, and the self. Journal of Reaserch in Personality, 29, 395-422.

Masten, A. (2001). Ordinary magic. Resilience processes in development. American Psychologist, 56(3), 227-238. http://dx.doi.org/10.1037/0003-066X.56.3.227

Masten, A. S., \& Reed, M. G. (2002). Resilience in development. In C. R. Snyder, \& S. J. Lopez (Eds.), Handbook of positive psychology (pp. 74-88). London, England: Oxford University Press.

Praissman, S. (2008). Mindfulness-based stress reduction: A literature review and clinician's guide. Journal of the American Academy of Nurse Practitioners, 20(4), 212-216. http://dx.doi.org/10.1111/j.1745-7599.2008.00306.x

Seif Naraghi, M. W., \& Naderi, A. (2011). Psychology and Education Exceptional Children. Tehran, Arasbaran.

Siegel, R. D. (2010). The mind fullness solution: Everyday practices for everyday problems. New York: Guilford.

Veer, S. D., Brouwers, A., Evers, W., \& Tomic, W. (2009). A pilot study of the psychological impact of the mindfulness-based stress reduction program on person who stutter. European Psychotherapy, 9(1), 39-56.

Zeidan, F., Gordon, N. S., Merchant, J., \& Goolkasian, P. (2010). The Effects of Brief Mindfulness Meditation Training on Experimentally Induced Pain. The Journal of Pain, 11(3), 199-209.

\section{Copyrights}

Copyright for this article is retained by the author, with first publication rights granted to the journal.

This is an open-access article distributed under the terms and conditions of the Creative Commons Attribution license (http://creativecommons.org/licenses/by/4.0/). 